Open Access

\title{
Brain-controlled functional electrical stimulation therapy for gait rehabilitation after stroke: a safety study
}

\author{
Colin M. McCrimmon ${ }^{1 *}$, Christine E. King ${ }^{1}$, Po T. Wang ${ }^{1}$, Steven C. Cramer ${ }^{2,3,4}$, Zoran Nenadic ${ }^{1,5^{*}}$ and An H. Do ${ }^{2}$
}

\begin{abstract}
Background: Many stroke survivors have significant long-term gait impairment, often involving foot drop. Current physiotherapies provide limited recovery. Orthoses substitute for ankle strength, but they provide no lasting therapeutic effect. Brain-computer interface (BCl)-controlled functional electrical stimulation (FES) is a novel rehabilitative approach that may generate permanent neurological improvements. This study explores the safety and feasibility of a foot-drop-targeted BCI-FES physiotherapy in chronic stroke survivors.

Methods: Subjects ( $n=9$ ) operated an electroencephalogram-based BCl-FES system for foot dorsiflexion in 12 one-hour sessions over four weeks. Gait speed, dorsiflexion active range of motion (AROM), six-minute walk distance (6MWD), and Fugl-Meyer leg motor (FM-LM) scores were assessed before, during, and after therapy. The primary safety outcome measure was the proportion of subjects that deteriorated in gait speed by $\geq 0.16 \mathrm{~m} / \mathrm{s}$ at one week or four weeks post-therapy. The secondary outcome measures were the proportion of subjects that experienced a clinically relevant decrease in dorsiflexion AROM $\left(\geq 2.5^{\circ}\right), 6 \mathrm{MWD}(\geq 20 \%)$, and FM-LM score $(\geq 10 \%)$ at either post-therapy assessment.
\end{abstract}

Results: No subjects (0/9) experienced a clinically significant deterioration in gait speed, dorsiflexion AROM, 6MWT distance, or FM-LM score at either post-therapy assessment. Five subjects demonstrated a detectable increase $(\geq 0.06 \mathrm{~m} / \mathrm{s})$ in gait speed, three subjects demonstrated a detectable increase $\left(\geq 2.5^{\circ}\right)$ in dorsiflexion AROM, five subjects demonstrated a detectable increase $(\geq 10 \%)$ in $6 \mathrm{MWD}$, and three subjects demonstrated a detectable increase $(\geq 10 \%)$ in FM-LM. Five of the six subjects that exhibited a detectable increase in either post-therapy gait speed or 6MWD also exhibited significant ( $p<0.01$ using a Mann-Whitney $U$ test) increases in electroencephalogram event-related synchronization/ desynchronization. Additionally, two subjects experienced a clinically important increase $(\geq 0.16 \mathrm{~m} / \mathrm{s})$ in gait speed, and four subjects experienced a clinically important increase $(\geq 20 \%)$ in 6MWD. Linear mixed models of gait speed, dorsiflexion AROM, 6MWD, and FM-LM scores suggest that BCI-FES therapy is associated with an increase in lower motor performance at a statistically, yet not clinically, significant level.

Conclusion: BCl-FES therapy is safe. If it is shown to improve post-stroke gait function in future studies, it could provide a new gait rehabilitation option for severely impaired patients. Formal clinical trials are warranted.

Keywords: Gait therapy, Stroke recovery, Brain computer interface, Electrical stimulation, EEG, Dorsiflexion

\footnotetext{
*Correspondence: cmccrimm@uci.edu; znenadic@uci.edu

${ }^{1}$ Department of Biomedical Engineering, University of California, Irvine, CA 92697, USA

Full list of author information is available at the end of the article
} 


\section{Introduction}

Strokes are the leading cause of long-term disability in the U.S. with over 795,000 new cases each year [1], a number that will grow as the population ages and stroke survival rates increase. Despite physiotherapy and spontaneous recovery, $\sim 2$ million stroke survivors in the U.S. suffer from long-term gait deficits. Post-stroke gait disability contributes to decreased participation in physical, social, and professional activities [2, 3], thereby exacerbating co-morbidities such as diabetes, cardiovascular disease, and depression [1].

The inability to dorsiflex the ankle during the swing phase of the gait cycle, known as foot drop, can contribute to gait problems, such as reduced walking speed $[4,5]$. Many studies have shown that the use of an ankle-foot orthosis, which specifically corrects foot drop, improves gait velocity in stroke survivors [6-9]. Currently available orthoses and assistive gait devices (e.g. walkers and functional electrical stimulation [FES] devices) have not been shown to provide lasting effects after removal $[10,11]$. Therefore, novel therapies that provide substantial, longterm gait improvement for stroke survivors are urgently needed.

There has been a growing interest in employing braincomputer interface (BCI) technology in post-stroke gait therapy [12-16]. BCIs use computers to translate signals of the central nervous system (e.g. via electroencephalography [EEG]) into control commands for external devices, providing peri-infarct areas with effector control in cortical strokes or bypassing the lesion in subcortical strokes. It has been hypothesized that by coupling the activation of upper motor neurons (UMNs) in the post-stroke cerebral cortex with the activation of $\alpha$ lower motor neurons (e.g. via functional electrical stimulation), lasting neurological and functional improvement may be achieved through a Hebbian learning process [17]. Utilizing a BCI ensures that patients are activating UMNs while receiving FES therapy, as opposed to passive (nonvolitional) electrical stimulation which may have less therapeutic potential [18]. Several reports on the implementation of braincontrolled FES systems [12-14] [19] and their application to stroke rehabilitation $[13,14,20]$ have emphasized its promise as a new physiotherapeutic modality. However, no studies have systematically assessed safety and efficacy with behavioral outcomes, an important step before largescale clinical trials can be performed. Although EEG and FES are quite safe separately, it is unknown whether they could promote maladaptive motor control and cause a deterioration in gait function when they are used in combination. For example, animal studies had shown that untimely coactivation of pre- and post-synaptic neurons can lead to long-term synaptic depression [21].

This Phase I clinical trial examined the safety of a novel foot-drop-targeted BCI-FES physiotherapy in a cohort of chronic stroke survivors using a previously developed system [12, 19]. Additionally, since this BCI-FES therapy may alleviate foot drop, and subsequently lead to an increased gait velocity [22], post-hoc analyses were performed to explore its potential efficacy and uncover any associated brain physiological changes.

\section{Methods \\ Study design}

The study was approved by the University of California, Irvine, Institutional Review Board. Qualified chronic stroke subjects participated in 12 sessions of BCI-FES therapy for foot drop. Each subject first underwent the following baseline assessments: fast gait speed, dorsiflexion active range of motion (AROM), six-minute walk distance (6MWD), and Fugl-Meyer leg motor (FM-LM) score. The BCI-FES therapy was then administered at a rate of three one-hour sessions per week over the course of four weeks. The system was designed to detect when the subject was trying to dorsiflex his/her paretic foot, using EEG, and deliver electrical stimulation to the appropriate deep peroneal nerve. Neurological and functional assessments were performed immediately prior to every third session, as well as one week and four weeks after the $12^{\text {th }}$ session. The schedule of activities is summarized in Fig. 1. A before-and-after comparison was used to determine if any of the outcome measures deteriorated significantly.

\section{Subject recruitment}

Advertisements were used to recruit stroke survivors ( $>6$ mo post-stroke) with gait impairment that included foot drop. The entry criteria were: (1) age $>18$, (2) ability to walk $\geq 10 \mathrm{~m}$ without the use of an ankle-foot orthosis or assistance by another person (walking aids, e.g. a cane or walker, were permitted but had to be used consistently), (3) sufficient cognitive and language function to follow study-related instructions reliably. The exclusion criteria were: (1) absence of a tibialis anterior response to FES, (2) inability to tolerate FES, (3) presence of electronic implants, (4) severe spasticity or plantarflexion contracture (Modified Ashworth scale $=4$ ). Although subjects were allowed to continue their personal exercise programs, initiating new rehabilitative treatments or physiotherapies during the study would result in exclusion from the study.

\section{BCI-FES dorsiflexion therapy}

Qualified subjects underwent 12 one-hour long sessions of the experimental BCI-FES dorsiflexion therapy, generally performed at a rate of three sessions per week across four weeks. Before each session, the subjects were fitted with an EEG cap (10-10 International Standard), and impedances were reduced to $<10 \mathrm{k} \Omega$ for a fixed 32-channel set. To provide the system with training EEG data, subjects followed 100 alternating six-second-long cues to relax or dorsiflex the paretic foot. Subjects were visually 


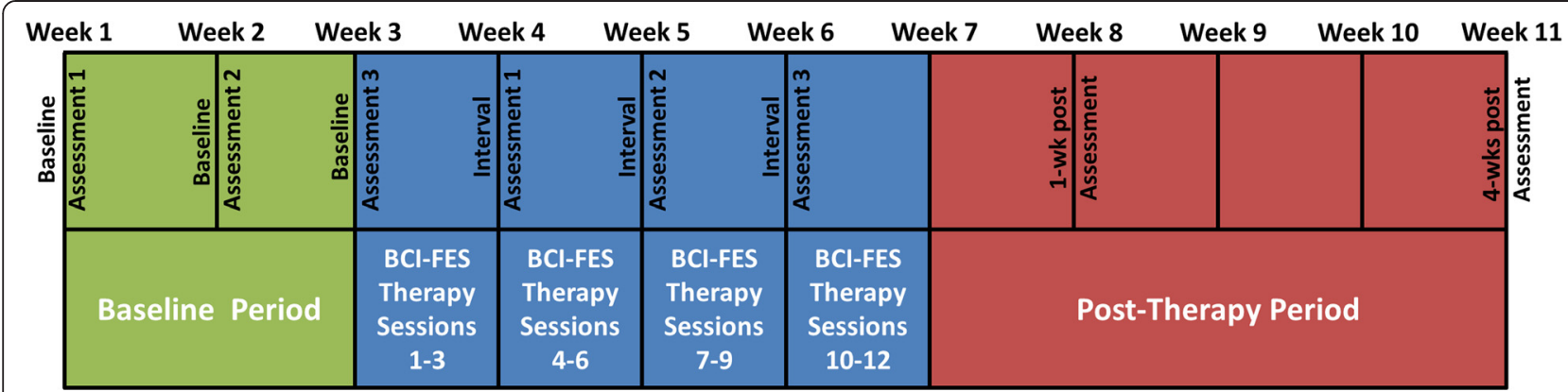

Fig. 1 Schedule of activities for each subject. Interval Assessments 1, 2, and 3 were performed immediately before BCI-FES therapy sessions 4, 7, and 10 , respectively

monitored for mirror movements (involuntary movements of a limb that are synchronous with the voluntary movements of the contralateral limb [23]) and cocontractions at other limbs, and were asked to discontinue these if they occurred. However, compliance with these instructions was not formally measured. The training data were recorded at $256 \mathrm{~Hz}$ with a NeXus-32 bioamplifier (Mind Media, Herten, Netherlands) to generate a session-specific decoding model for online operation (methods in [24-26]). This model could distinguish idling from dorsiflexion using EEG. Finally, surface FES electrodes were placed over the proximal course of the deep peroneal nerve (paretic side only), and stimulation parameters were adjusted to achieve $\sim 15^{\circ}$ dorsiflexion from the neutral position without discomfort.

During each one-hour-long session, subjects performed as many online BCI-FES runs as possible. Each run consisted of 10 alternating, contiguous 10-s-long dorsiflex/ relax cues, during which the BCI-FES system detected the subjects' intention to dorsiflex (or relax) from EEG and correspondingly provided (or withheld) stimulation. The accuracy of BCI operation was calculated as the percentage of correctly determined BCI-states at a rate of four decisions per second (decisions were calculated every $0.25 \mathrm{~s}$ based on the most recent $0.75 \mathrm{~s}$ of EEG data [24]).

Subjects were informed of the possibility of erroneous recognition of the idle and dorsiflexion states by the BCI. If FES was erroneously delivered when no movement was intended, subjects were instructed to ignore the stimulation and continue to relax; when movement was intended but no FES was delivered, subjects were instructed to continue to attempt dorsiflexion. Mirror movements and co-contractions were monitored visually. Brief breaks were provided between runs, or when requested, to prevent fatigue of the deep peroneal nerve and tibialis anterior muscle.

\section{Neurological and functional assessments}

Neurological and functional measurements (fast gait speed, dorsiflexion AROM, 6MWD, and FM-LM score) were performed before, during, and after the BCI-FES therapy, and are defined below:

- Gait Speed: Fast gait speed [27] was measured [28] for the middle 6-m section of a 10-m walkway. This test was repeated 5 times at each assessment, and the average speed was calculated [29]. AFOs were removed, but walking aids such as walkers and canes were allowed. If a subject did use a walking aid at their first baseline assessment, they were asked to continue to use the same device throughout the rest of the study.

- Dorsiflexion AROM: The subject was placed in a seated position with the knee flexed at $90^{\circ}$ and the tibial shank perpendicular to the ground. A goniometer was used to measure the AROM at the ankle as the subject dorsiflexed, using standard technique [30].

- 6 min Walk Distance: Assessed as the distance that subjects can ambulate (at a safe, casual speed) in 6 min [31]. AFOs were removed, but walking aids were permitted.

- Fugl-Meyer Leg Motor Score: Assessed using the FM measurement system as defined in [32, 33].

Although chronic stroke subjects are assumed to have reached a plateau in terms of spontaneous behavioral recovery [34, 35], three baseline assessments (see Fig. 1) were performed to account for day-to-day variance [36]. Assessments were also performed immediately prior to every third BCI-FES session and again one week and four weeks after the $12^{\text {th }}$ session. Subjects were also asked to maintain a fall diary throughout the study. The results of this diary were documented at each weekly functional assessment.

\section{Outcome measures}

All pre-stated outcome measures focused on safety. The primary outcome was the proportion of subjects who 
demonstrated a deterioration in gait speed $\geq 0.16 \mathrm{~m} / \mathrm{s}$ at either the one-week or four-weeks post-therapy assessment. This threshold was chosen as it may be associated with a change in the modified Rankin Scale (mRS) for the post-stroke population [37]. The secondary outcome measures included the proportion of subjects who experienced a significant deterioration in dorsiflexion AROM, 6MWD, and FM-LM score at either the one-week or four-weeks post-therapy assessment. Deterioration of dorsiflexion AROM is defined as a $\geq 2.5^{\circ}$ decrease from average baseline. There is no established minimum clinically important change in dorsiflexion AROM, so this threshold was chosen as it represents the minimal detectable difference [38]. The minimum clinically significant change in 6MWD is $20 \%$ [39], while that of the FM-LM score is hypothesized to be $10 \%$ [40].

\section{Post-Hoc analyses}

Additional analyses were performed to determine the proportion of subjects who demonstrated a detectable increase in gait speed $(\geq 0.06 \mathrm{~m} / \mathrm{s}[37,39])$, dorsiflexion $\operatorname{AROM}\left(\geq 2.5^{\circ}\right), 6 \mathrm{MWD}(\geq 10 \%[41])$, and FM-LM score $(\geq 10 \%)$ from average baseline at both post-therapy assessments. Detectable changes are not necessarily clinically important, so the proportion of subjects that experienced a clinically significant increase in gait speed $(\geq 0.16 \mathrm{~m} / \mathrm{s})$ and 6MWD $(\geq 20 \%)$ was also calculated. Furthermore, the effect of BCI-FES therapy on gait speed, dorsiflexion AROM, 6MWD, and FM-LM was determined using independent linear mixed models (LMMs) with outcomes (gait speed, etc.) as a function of therapy group (pretherapy baseline or post-therapy) with the random effects being subjects (and their interaction with therapy group) and repetitions within subjects (and their interaction with therapy group).

The training EEG datasets were analyzed for each session to determine if subjects experienced any brain changes throughout the course of the therapy. To reveal any spatial changes associated with therapy, the dorsiflexion-related importance (the $\mu$-measure defined in [42]) of each EEG channel was plotted for all training sessions (details in Additional file 1). Additionally, changes in event-related synchronization (ERS) and event-related desynchronization (ERD) throughout the therapy were analyzed as follows. The most important channel (highest average $\mu$ across all sessions) was identified for each subject, and the EEG data from this channel were aggregated by week. The median ERS and ERD at each frequency $(8-30 \mathrm{~Hz}$ in $2 \mathrm{~Hz}$ bins) were calculated for weeks two through four (Equations 2 and 3 in Additional file 1) and compared to their respective week-one value using repeated Mann-Whitney $U$ tests (Bonferroni corrected $\alpha=0.01$ ).

\section{Results}

\section{Overview}

Nine subjects provided their informed consent to participate in the study (Table 1). All subjects completed the baseline and interval assessments, 12 sessions of BCI-FES therapy, and the one-week post-therapy assessment. Subject S1 suffered a recurrent stroke after the one-week post-therapy assessment, and therefore the four-weeks post-therapy assessment could not be obtained. Subject S2 could not be contacted for the fourweeks post-therapy assessment. Subject S6 experienced leg pain during Interval Assessment 2 and declined the 6MWD test. The subjects averaged 8.4 BCI-FES runs per session. Almost $95 \%$ of the total number of BCI-FES runs across all subjects and sessions were at a significant performance level $(\alpha=0.01)$ compared to MonteCarlo simulation (details in [24]; see Table 1).

\section{Primary outcome measure}

No subjects demonstrated a clinically significant decrement in gait speed at either the one-week (subjects S1-S9) or the four-weeks (subjects S3-S9) post-therapy assessment (see Fig. 2). The test-retest reliability [43, 44] of the gait speed measurements, $>0.98$, was comparable to reported values $[41,45]$. Post-hoc analysis revealed that five out of nine subjects (56\%) exhibited a detectable increase in gait speed at both post-therapy assessments. S9 experienced a detectable increase in gait speed at one week post-therapy that disappeared by the last assessment. S6 experienced a detectable increase in gait speed only at four weeks post-therapy. Additionally, two of nine subjects (22\%) experienced a clinically important increase in gait speed $(\geq 0.16 \mathrm{~m} / \mathrm{s})$ at both post-therapy assessments. S6 experienced a clinically important increase in gait speed only at four weeks post-therapy. Note that although these increases may be clinically relevant, they are not necessarily statistically significant. With a pre-post therapy slope of $0.025(p \leq 0.03$; intercept $=0.63)$, the LMM demonstrated that BCI-FES therapy may be associated with an increase in gait speed.

\section{Secondary outcome measures}

No subjects experienced a deterioration in dorsiflexion AROM, 6MWD, or FM-LM score at either the one-week or four-weeks post-therapy assessment (Figs. 3, 4 and 5). Post-hoc analysis revealed that three out of nine subjects (33\%) had a detectable $\left(\geq 2.5^{\circ}\right)$ increase in dorsiflexion AROM at both post-therapy assessments. Five subjects (out of nine) had detectable increase by the first posttherapy assessment, while only two subjects (out of seven) retained this increase at four weeks post-therapy. With a pre-post therapy slope of $0.84(p=0.013$; intercept $=4.22)$, the LMM demonstrated that BCI-FES therapy may be associated with an increase in dorsiflexion AROM. The 
Table 1 Demographic and $\mathrm{BCl}$ performance data for all subjects

\begin{tabular}{|c|c|c|c|c|c|c|c|c|c|c|c|c|c|}
\hline Subject & Age/Sex & $\begin{array}{l}\text { Time Since } \\
\text { Stroke (mo) }\end{array}$ & $\begin{array}{l}\text { Stroke } \\
\text { Type }\end{array}$ & Stroke Location & Clinical Presentation & $\begin{array}{l}\text { NIH } \\
\text { Stroke } \\
\text { Scale }\end{array}$ & $\begin{array}{l}\text { Barthel } \\
\text { Index }\end{array}$ & $\begin{array}{l}\text { Geriatric } \\
\text { Depress } \\
\text { ion Score }\end{array}$ & $\begin{array}{l}\text { FM-LM Score } \\
\text { (first/last } \\
\text { assessm ent) }\end{array}$ & $\begin{array}{l}\text { Walking Aids } \\
\text { Used at Time } \\
\text { of Study }\end{array}$ & $\begin{array}{l}\text { Previous } \\
\text { Lower Limb } \\
\text { FES Use }\end{array}$ & $\begin{array}{l}\text { BCl-NMES Runs } \\
\text { Completed }^{\mathrm{a}}\end{array}$ & $\begin{array}{l}\text { Overall Decoding } \\
\text { Accuracy (\%) }\end{array}$ \\
\hline S1 & $83 / M$ & 29 & I & L basal ganglia & $\mathrm{R}$ hemiparesis & 5 & 90 & 0 & $23 / 27$ & AFO & N & 107 & 82.7 \\
\hline S2 & $59 / F$ & 9 & I & R internal capsule & L hemiplegia & 8 & 60 & 4 & $21 / 25$ & $\mathrm{AFO}+\mathrm{C}$ & N & 98 & 79.1 \\
\hline S3 & $35 / M$ & 24 & $\mathrm{H}$ & $\mathrm{R}$ corona radiata & L hemiparesis & 6 & 100 & 0 & $29 / 31$ & AFO & Y & 119 & 61.6 \\
\hline S4 & $75 / \mathrm{M}$ & 9 & I & $\begin{array}{l}\text { L putamen/corona } \\
\text { radiata }\end{array}$ & $\mathrm{R}$ hemiparesis & 7 & 75 & 7 & $18 / 21$ & $\mathrm{AFO}+\mathrm{W}$ & N & 111 & 74.5 \\
\hline S5 & $51 / \mathrm{M}$ & 8 & I & L basal ganglia & R foot drop & 3 & 100 & 6 & $25 / 26$ & AFO & N & 113 & 84.2 \\
\hline S6 & $71 / M$ & 102 & । & $\mathrm{R}$ basal ganglia & L hemiparesis & 2 & 100 & 1 & $25 / 29$ & AFO & N & 97 & 75.5 \\
\hline S7 & $66 / F$ & 23 & । & L pons & $\mathrm{R}$ hemiparesis & 5 & 95 & 4 & $21 / 26$ & $\mathrm{AFO}+\mathrm{C}$ & N & 103 & 86.9 \\
\hline S8 & $38 / F$ & 24 & $\mathrm{H}$ & R basal ganglia & $\mathrm{L}$ hemiparesis & 5 & 70 & 1 & $23 / 22$ & $\mathrm{AFO}+\mathrm{C}$ & N & 80 & 84.1 \\
\hline S9 & $60 / M$ & 19 & $\mathrm{H}$ & R thalamus & L hemiparesis & 5 & 100 & 2 & $26 / 26$ & AFO & N & 82 & 88.6 \\
\hline
\end{tabular}

M: male, F: female, l: ischemic, $H$ : hemorrhagic, L: left, $R$ : right AFO: ankle-foot orthosis, C: cane, W: walker, $Y:$ yes, N: no

${ }^{\mathrm{a}}$ Out of a total of 910 runs, 864 were deemed significant $(a=0.01)$ by comparison to Monte-Carlo Simulation 

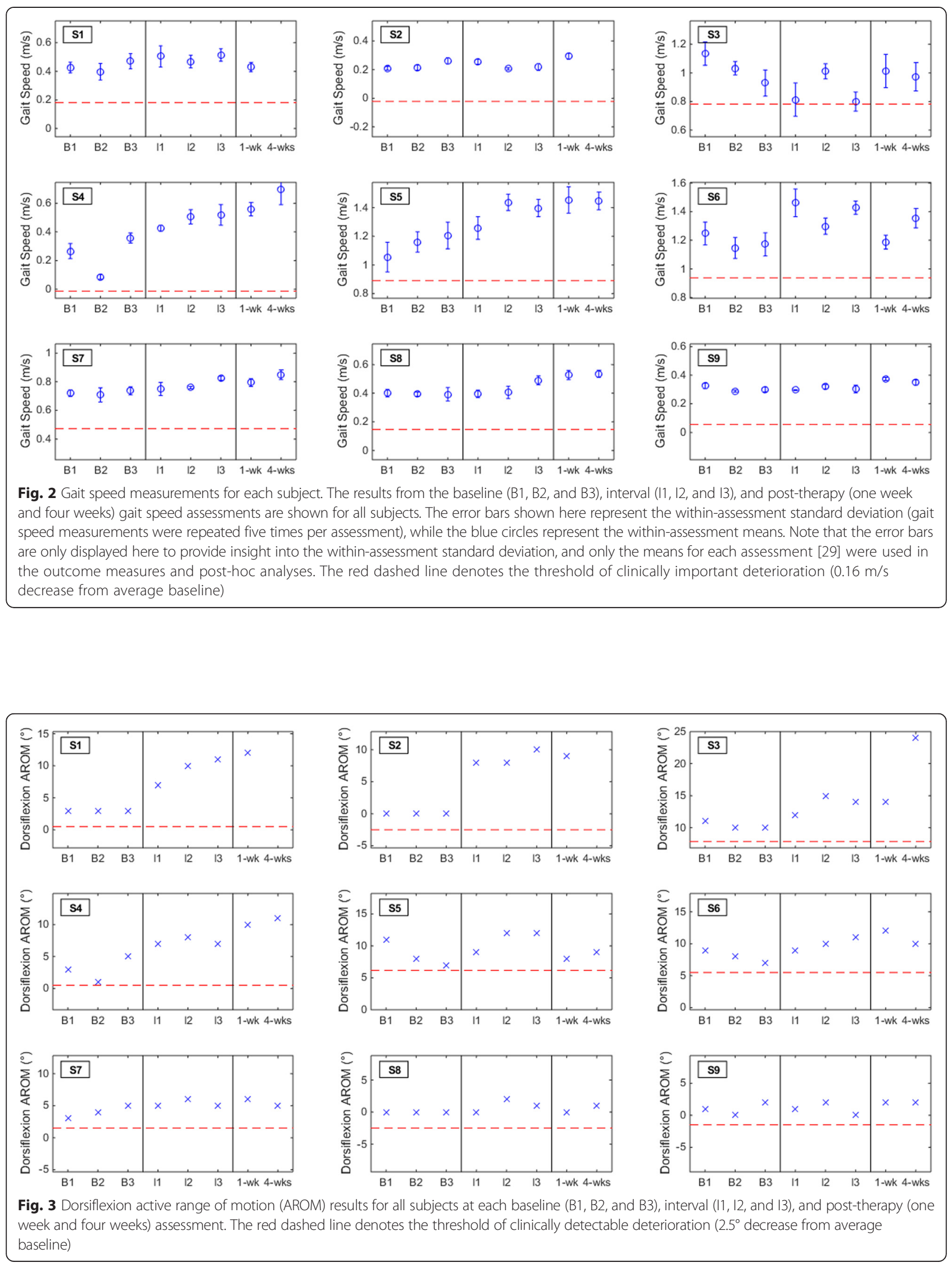

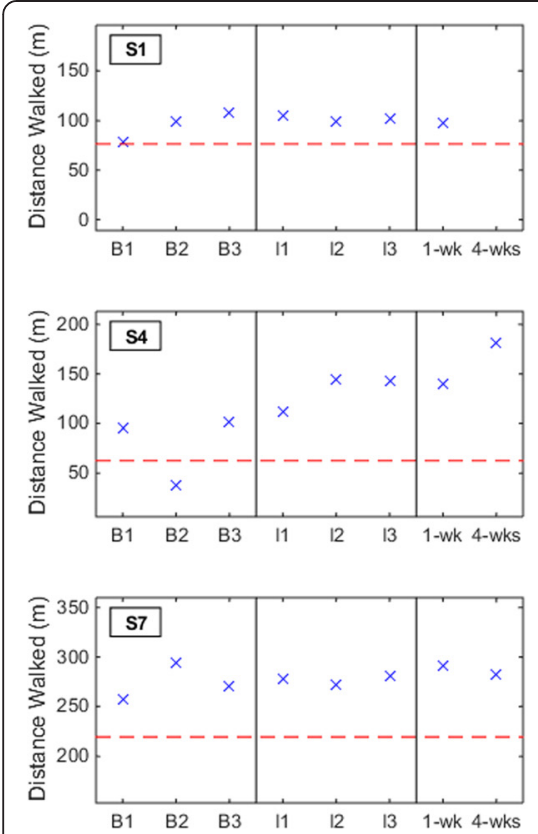
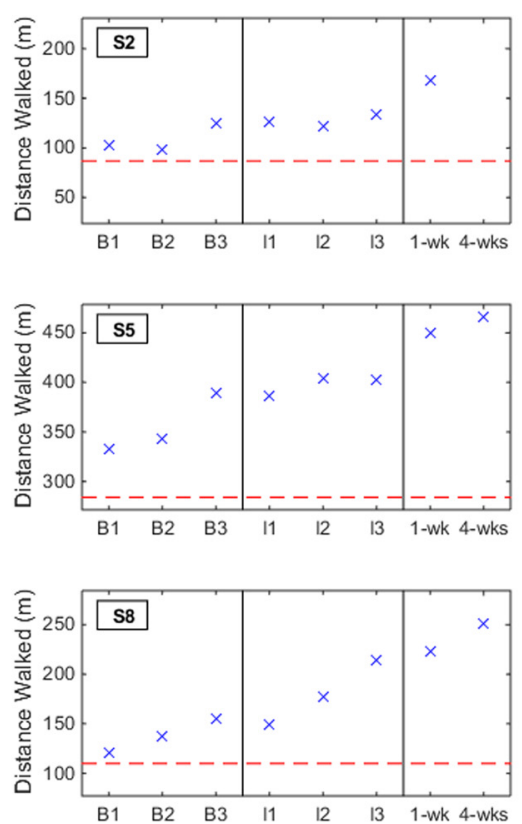
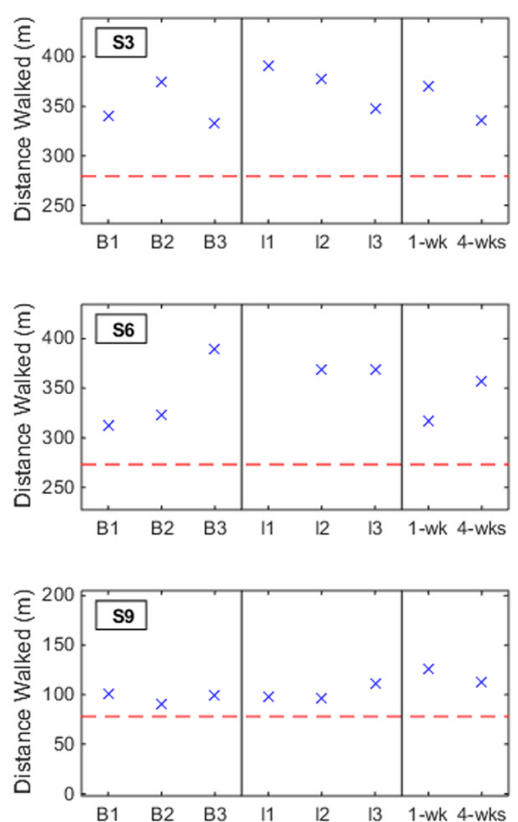

Fig. 4 Subjects' six minute walking distance (6MWD) at each baseline (B1, B2, and B3), interval (I1, I2, and I3), and post-therapy (one week and four weeks) assessment. The red dashed line denotes the threshold of clinically important deterioration (20\% decrease from average baseline)

6MWD increased by $\geq 10 \%$ from average baseline for five subjects (56\%) and $\geq 20 \%$ for four subjects (44\%) at the post-therapy assessments. S9 experienced a detectable increase in 6MWD at both post-therapy assessments, but only the increase at one week post-therapy was also clinically important. With a pre-post therapy slope of 8.52
( $p=0.02$; intercept $=204.10)$, the LMM demonstrated that BCI-FES therapy may be associated with an increase in $6 M W D$. FM-LM score increased by $\geq 10 \%$ for subjects $\mathrm{S} 1$, S2, and S7 (33\% of subjects) at the post-therapy assessments. S6 only experienced an increase in FM-LM at the four-weeks post-therapy assessment. Once again, changes
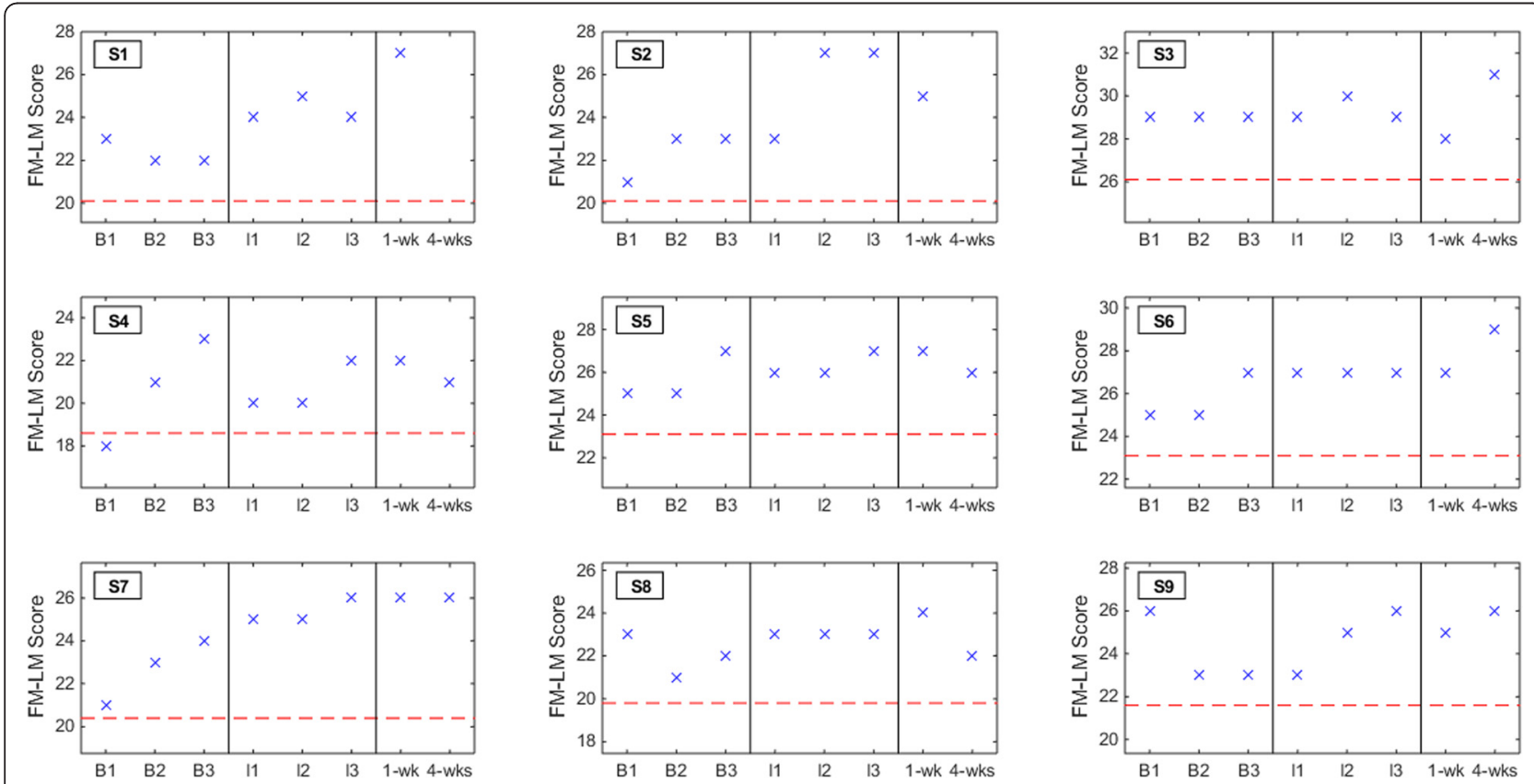

Fig. 5 Subjects' Fugl-Meyer leg motor (FM-LM) scores at each baseline (B1, B2, and B3), interval (I1, I2, and I3), and post-therapy (one week and four weeks) assessment. The red dashed line denotes the threshold of clinically detectable/important deterioration (10 \% decrease from average baseline) 
in dorsiflexion AROM, 6MWD, and FM-LM scores per subject may be clinically significant, but they are not necessarily statistically significant. With a pre-post therapy slope of $0.36(p<0.001$; intercept $=23.81)$, the LMM demonstrated that BCI-FES therapy may be associated with an increase in FM-LM score.

\section{Adverse events}

Subject S1 reported a recurrent stroke after his one-week post-therapy assessment. The cause of stroke was idiopathic, but was suspected to be associated with a prosthetic heart valve, and was thereby deemed unrelated to the BCI-FES therapy. Subject S7 reported a fall (not resulting in serious injury) while carrying a heavy object after the one-week post-therapy assessment. This was also considered unrelated to the study procedures. No other adverse events, such as peroneal nerve dysesthesias or skin breakdown, were reported.

\section{EEG changes during therapy}

Five out of the six subjects that exhibited a detectable improvement in post-therapy gait (increase in gait speed or 6MWD) also exhibited a significant increase in ERS and ERD (83.3 \% sensitivity). The remaining three subjects demonstrated no significant increase in ERS or ERD (100\% specificity). The evolution of ERS and ERD are shown in Fig. 6.

For subjects S1, S2, S4, S5, S6, S7, and S8, the most salient EEG channel for distinguishing idling from dorsiflexion (highest $\mu$ value) was $\mathrm{Cz}$; for S3 and S9, it was C5 and $\mathrm{CPz}$, respectively. Representative maps of the EEG channels' importance for dorsiflexion are shown in Fig. 7 (additional plots in Additional file 1). No subject demonstrated a consistent change in the location of his/her most salient EEG channel throughout the BCI-FES therapy.

\section{Subjective reports}

Subject S2 indicated at the one-week post-therapy assessment that she could climb stairs for the first time since her stroke. Subject S4 indicated that his newfound gait improvement allowed him to walk farther during his daily exercises. Subject S5 stated that he had better control of the gas pedal while driving after beginning the BCI-FES therapy. Although subject S6 felt that the therapy was somewhat tedious, he was enthusiastic about his improvement in dorsiflexion AROM. Subject S7 felt that she had more consistent control of foot dorsiflexion and knee flexion/extension. Subject S9 reported an increased sensation and strength in his paretic side, leading to what he considered to be a more natural gait. All reports were provided or solicited informally at the end of the study.

\section{Discussion}

No clinically important deterioration in gait speed, dorsiflexion AROM, 6MWT distance, or FM-LM score was present one week or four weeks after completing the BCI-FES therapy. Throughout the study, only one fall was reported, an incidence well below that of conventional outpatient stroke rehabilitation [46-48]. The lack of deterioration in the measured gait characteristics indicates that the BCI-FES dorsiflexion therapy may be safe to explore in larger populations of stroke survivors. In addition, post-hoc analysis revealed that five subjects were walking detectably faster $(\geq 0.06 \mathrm{~m} / \mathrm{s})$ at the post-therapy assessments. Two of these subjects were walking $\geq 0.16 \mathrm{~m} / \mathrm{s}$ faster than average baseline, quite a remarkable feat since this magnitude is associated with a $\geq 1$ increase in mRS [37]. Furthermore, the data suggest that BCI-FES therapy is associated with a statistically significant, albeit not clinically significant, increase in gait speed, dorsiflexion AROM, 6MWD, and FM-LM score. Since this study focused on safety outcomes and was not designed to test efficacy, any improvement in gait function cannot yet be attributed to the BCI-FES therapy. Nonetheless, given that increased gait speed is strongly associated with increased social re-integration after stroke [2], and based on this therapy's acceptable early safety profile, larger clinical trials are warranted to definitively establish its safety and efficacy.

Given that the BCI-NMES system was inspired by the concept of Hebbian learning between M1 and foot dorsiflexion motor pools [17], increased dorsiflexion AROM and associated brain changes were expected. However, increased dorsiflexion AROM generally did not persist at four weeks post-therapy (verified via an insignificant LMM slope using post-therapy data from only this assessment), and ERS and ERD changes occurred, instead, in association with increased gait speed alone. These increases in ERS and ERD indicate that activation of foot and leg sensorimotor areas is more synchronous (Fig. 7 and Additional file 1), and suggest the presence of an underlying neural process. Possible mechanisms include Hebbian learning between UMNs and the spinal cord gait central pattern generators [49] and increased afferent sensory feedback during electrical stimulation and walking [50]. It also remains a possibility that modest increases in dorsiflexion strength (via the Hebbian learning mechanism expected by the authors) may have occurred, but subsequent increases in dorsiflexion AROM were obscured by mild plantar flexion contractures. Ultimately, formal physiological studies are needed to further elucidate the underlying mechanism.

Of note, the authors believe that subject S6 experienced problems with walking during the one-week post-therapy assessment due to a new pair of poorly 


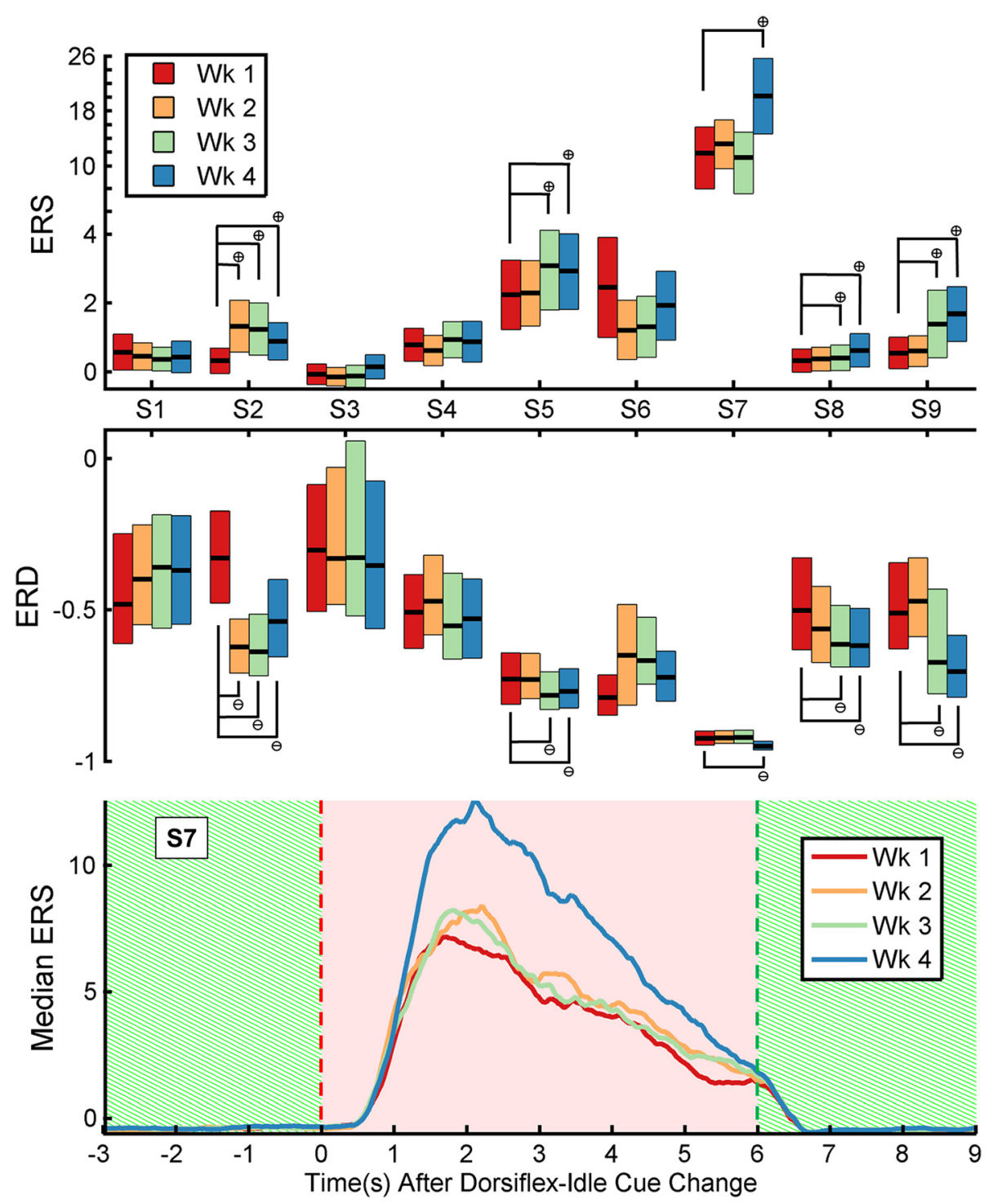

Fig. 6 Top and Middle: Event-related synchronization (ERS) and desynchronization (ERD) during each week for all subjects. ERS, top, and ERD, middle, during weeks one through four (wk 1-4, or sessions 1-3, 4-6, 7-9, 10-12, respectively). Boxes signify intervals of one median absolute deviation around the median (central black bar). Significant positive and negative changes from week one are denoted by a cross-in-circle symbol and a line-in-circle symbol, respectively. Bottom: Temporal profile of median ERS during weeks one through four, taken from S7. The cue being presented is denoted by the background ("Dorsiflex", hatched green; "Relax", solid red)

fitting shoes. This is supported by the presence of a transient drop in his gait speed and 6MWD. By four weeks post-therapy, these measures surpassed their baseline values.

Lastly, at the start of the study the mean baseline gait speed and 6MWD were $0.63 \pm 0.40 \mathrm{~m} / \mathrm{s}$ and $204.1 \pm$ $122.5 \mathrm{~m}$, respectively, lower than that of the chronic stroke population reported in [51]. One potential strength of BCI-based movement therapies is that they are accessible to individuals with baseline motor functions too low for other interventions (e.g. treadmill training).

\section{Limitations}

The major limitation of this study is the small sample size and the lack of matched controls and unbiased raters. Additionally, large, controlled studies, i.e. Phase III clinical trials, are necessary to definitively establish efficacy. However, the small sample size is appropriate for an initial investigation into the safety of this BCI-FES therapy. Furthermore, all subjects were assumed to have reached a steady rehabilitative state ( $>6$ months post-stroke) [34], and were thus used as their own controls. Also, baseline assessments for all subjects appeared steady. Since the 


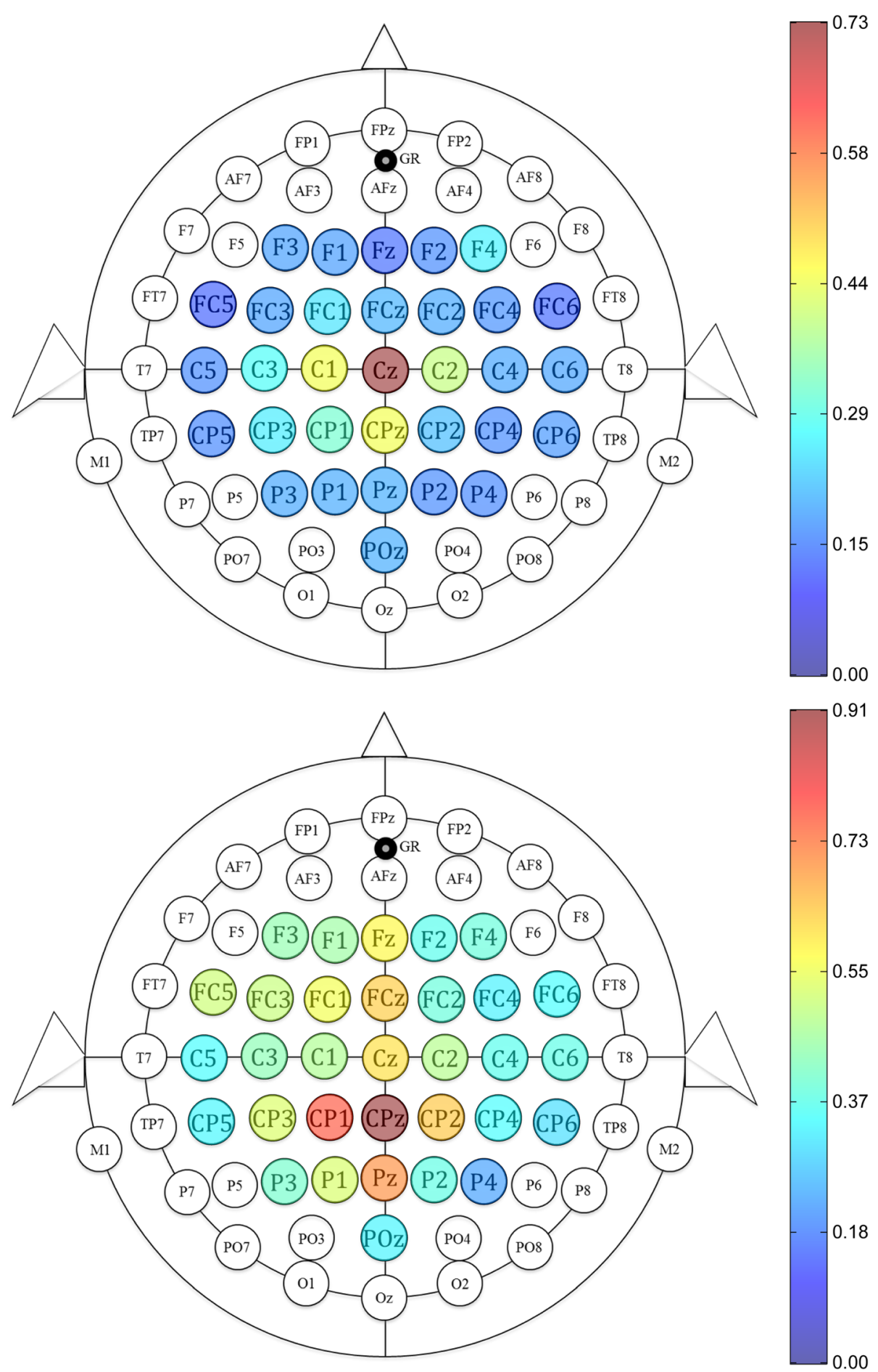

Fig. 7 Importance of each EEG channel for dorsiflexion. Channels are colored based on their $\mu$ value, from dark blue (unimportant) to dark red (highly important). These two representative maps are taken from subject S8 (top) and S9 (bottom)

majority of subjects had subcortical strokes, particularly in the basal ganglia, it will be important to examine how BCI-FES therapy affects those with cortical strokes in future studies. Ankle dorsiflexion was chosen as the target of FES therapy since studies have concluded that foot drop plays a role in post-stroke gait impairment $[4,5]$. On the other hand, studies such as [52] stress the important role of other muscle groups in post-stroke gait impairment. However, the BCI-FES therapy implemented in this study can easily be applied to other muscle groups. Finally, 
although multiple meta-analyses $[10,11]$ suggest that functional electrical stimulation alone does not provide a conclusive long-term therapeutic effect, a formal direct comparison with BCI-FES therapy may be necessary in the future.

\section{Conclusions}

Since this BCI-FES therapy appears to be safe and a large proportion of subjects experienced improvements in gait speed and dorsiflexion AROM, larger controlled studies (including pre-clinical and Phase II/III studies) are warranted to investigate additional aspects of this novel therapy. These include: (1) the potential efficacy and optimal duration of BCI-FES therapy, (2) the neurobiological principles that underlie any functional changes, (3) the subpopulation of stroke patients that will benefit most, and (4) any synergism with conventional physiotherapies.

\section{Additional file}

Additional file 1: Supplementary material and figures.

\section{Competing interests}

CEK received salary from HRL Laboratories (Malibu, California, USA). SCC has served as a consultant to GlaxoSmithKline, MicroTransponder, and Dart Neuroscience, and is a cofounder of personalRN. The remaining authors declare no competing interests.

\section{Authors' contributions}

CMM and CEK conducted the experiments. CMM also performed the data analyses and wrote the manuscript. PTW implemented the $\mathrm{BCl}$ software and provided technical support. SCC oversaw the study and helped with the manuscript. ZN designed and oversaw the study, developed the signal processing algorithm, assisted with the experiments, and helped with the manuscript. AHD designed and oversaw the study, recruited subjects, assisted with experiments, and helped with the manuscript. All authors read and approved the manuscript.

\section{Acknowledgements}

The authors acknowledge Renee Augsburger, MHA, C/NDT, Vicky Chan, MSPT, and Lucy Dodakian, MA, OTR/L, for their assistance in subject recruitment.

\section{Funding}

The study was supported by the American Academy of Neurology American Brain Foundation. The project was also supported by the National Science Foundation, through award 1160200, and by the National Center for Research Resources and the National Center for Advancing Translational Sciences, National Institutes of Health (NIH), through grant UL1 TR000153. CMM received support from the Medical Scientist Training Program at the University of California, Irvine. SCC acknowledges support from NIH grant K24 HD074722.

\section{Author details}

${ }^{1}$ Department of Biomedical Engineering, University of California, Irvine, CA 92697, USA. ²Department of Neurology, University of California, Irvine, Orange, CA 92868, USA. ${ }^{3}$ Department of Anatomy \& Neurology, University of California, Irvine, CA 92697, USA. ${ }^{4}$ Department of Physical Medicine \& Rehabilitation, University of California, Irvine, Orange, CA 92868, USA. ${ }^{5}$ Department of Electrical Engineering and Computer Science, University of California, Irvine, CA 92697, USA.

Received: 18 February 2015 Accepted: 23 June 2015

Published online: 11 July 2015

\section{References}

1. Roger VL, Go AS, Lloyd-Jones DM, Benjamin EJ, Berry JD, Borden WB, et al. Heart disease and stroke statistics-2012 update: a report from the American Heart Association. Circulation. 2012;125:e2-e220.

2. Lord SE, McPherson K, McNaughton HK, Rochester L, Weatherall M. Community ambulation after stroke: how important and obtainable is it and what measures appear predictive? Arch Phys Med Rehab. 2004;85:234-9.

3. Cramer SC. Improving outcomes after stroke by LEAPS (Locomotor Experience Applied Post-Stroke) and bounds. Stroke. 2011;42:3659-60.

4. Wade DT, Wood VA, Heller A, Maggs J, Langton HR. Walking after stroke. Measurement and recovery over the first 3 months. Scand J Rehab Med. 1987;19:25-30

5. Dorsch S, Ada L, Canning CG, Al-Zharani M, Dean C. The strength of the ankle dorsiflexors has a significant contribution to walking speed in people who can walk independently after stroke: an observational study. Arch Phys Med Rehab. 2012;93:1072-6.

6. Ferreira $L A B$, Neto HP, Grecco LAC, Christovão $T C L$, Duarte NAC, Lazzari RD, et al. Effect of Ankle-foot Orthosis on Gait Velocity and Cadence of Stroke Patients: A Systematic Review. J Phys Ther Sci. 2013;25:1503-8.

7. Leung J, Moseley A. Impact of Ankle-foot Orthoses on Gait and Leg Muscle Activity in Adults with Hemiplegia: Systematic literature review. Physiotherapy. 2003;89:39-55.

8. de Wit DC, Buurke JH, Nijlant JM, ljzerman MJ, Hermens HJ. The effect of an ankle-foot orthosis on walking ability in chronic stroke patients: a randomized controlled trial. Clin Rehabil. 2004;18:550-7.

9. Kluding PM, Dunning K, O'Dell MW, Wu SS, Ginosian J, Feld J, et al. Foot drop stimulation versus ankle foot orthosis after stroke: 30-week outcomes. Stroke. 2013;44:1660-9.

10. Langhorne P, Coupar F, Pollock A. Motor recovery after stroke: a systematic review. Lancet Neurol. 2009:8:741-54.

11. Van Peppen RPS, Kwakkel G, Wood-Dauphinee S, Hendriks HJM, Van der Wees PJ, Dekker J. The impact of physical therapy on functional outcomes after stroke: what's the evidence? Clin Rehab. 2004;18:833-62.

12. Do AH, Wang PT, King CE, Schombs A, Cramer SC, Nenadic Z. Brain-computer interface controlled functional electrical stimulation device for foot drop due to stroke. In: Proc 34th Ann Int Conf IEEE EMBS. 2012. p. 6414-7.

13. Daly JJ, Cheng R, Rogers J, Litinas K, Hrovat K, Dohring M. Feasibility of a new application of noninvasive brain computer interface $(\mathrm{BCl})$ : a case study of training for recovery of volitional motor control after stroke. J Neurol Phys Ther. 2009;33:203-11.

14. Takahashi M, Takeda K, Otaka Y, Osu R, Hanakawa T, Gouko M, Ito K. Event related desynchronization-modulated functional electrical stimulation system for stroke rehabilitation: a feasibility study. J Neuroeng Rehabil. 2012;9:56.

15. Ramos-Murguialday A, Broetz D, Rea M, Läer L, Yilmaz Ö, Brasil FL, et al. Brain-machine interface in chronic stroke rehabilitation: a controlled study. Ann Neurol. 2013;74:100-8.

16. McGie SC, Zariffa J, Popovic MR, Nagai MK. Short-term neuroplastic effects of brain-controlled and muscle-controlled electrical stimulation. Neuromodulation. 2015:18:233-40.

17. Rushton DN. Functional electrical stimulation and rehabilitation-an hypothesis. Med Eng Phys. 2003;25:75-8.

18. Barsi Gl, Popovic DB, Tarkka IM, Sinkjaer T, Grey MJ. Cortical excitability changes following grasping exercise augmented with electrical stimulation. Exp Brain Res. 2008;191:57-66.

19. Do AH, Wang PT, King CE, Abiri A, Nenadic Z. Brain-computer interface controlled functional electrical stimulation system for ankle movement. J Neuroeng Rehabil. 2011:8:49.

20. Mukaino M, Ono T, Shindo K, et al. Efficacy of brain-computer interface-driven neuromuscular electrical stimulation for chronic paresis after stroke. J Rehabil Med. 2014;46:378-82.

21. Jacob V, Brasier DJ, Erchova I, Feldman D, Shulz DE. Spike Timing-Dependent Synaptic Depression in the In Vivo Barrel Cortex of the Rat. J Neurosci. 2007;27:1271-84.

22. Mueller MJ, Minor SD, Schaaf JA, Strube MJ, Sahrmann SA. Relationship of Relationship of Plantar-Flexor Peak Torque and Dorsiflexion Range of Motion to Kinetic Variables During Walking. Phys Ther. 1995;75:684-93.

23. Nelles G, Cramer SC, Schaechter JD, Kaplan JD, Finklestein SP. Quantitative assessment of mirror movements after stroke. Stroke. 1998;29:1182-7.

24. McCrimmon CM, King CE, Wang PT, Cramer SC, Nenadic Z, Do AH. Braincontrolled functional electrical stimulation for lower-limb motor recovery in stroke survivors. Proc 36th Ann Int Conf IEEE EMBS. 2014. p. 1247-50. 
25. Das K, Nenadic Z. Approximate information discriminant analysis: a computationally simple heteroscedastic feature extraction technique. Pattern Recogn. 2008;41:1548-57.

26. Das K, Nenadic Z. An efficient discriminant-based solution for small sample size problem. Pattern Recogn. 2009;42:857-66.

27. Bohannon RW. Comfortable and maximum walking speed of adults aged 20-79 years: reference values and determinants. Age Ageing. 1997;26:15-9.

28. Perry J, Burnfield JM. Gait Analysis-Normal and Pathological Function. 2nd ed. Thorofare, NJ, USA: Slack Incorporated; 2010.

29. Watson MJ. Refining the Ten-metre Walking Test for Use with Neurologically Impaired People. Physiotherapy. 2002;88:386-97.

30. Floyd RT, Thompson C. Manual of Structural Kinesiology. McGraw-Hill Education; 2011.

31. Guyatt GH, Sullivan MJ, Thompson PJ, Fallen EL, Pugsley SO, Taylor DW, et al. The 6-min walk: a new measure of exercise capacity in patients with chronic heart failure. Can Med Assoc J. 1985;132:919.

32. Fugl-Meyer A, Jaasko L, Leyman I, Olsson S, Steglind S. The post-stroke hemiplegic patient. 1. a method for evaluation of physical performance. Scand J Rehab Med. 1974;7:13-31.

33. See J, Dodakian L, Chou C, Chan V, McKenzie A, Reinkensmeyer DJ, et al. A standardized approach to the Fugl-Meyer assessment and its implications for clinical trials. Neurorehab Neural Re. 2013;27:732-41.

34. Duncan PW, Lai SM. Stroke recovery. Top Stroke Rehabil. 1997;4:51-8.

35. Skilbeck CE, Wade DT, Hewer RL, Wood VA. Recovery after stroke. J Neurol Neurosur Ps. 1983:46:5-8

36. Green J, Forster A, Young J. Reliability of gait speed measured by a timed walking test in patients one year after stroke. Clin Rehabil. 2002;16:306-14.

37. Tilson JK, Sullivan KJ, Cen SY, Rose DK, Koradia CH, Azen SP, et al. Locomotor Experience Applied Post Stroke (LEAPS) Investigative Team. Meaningful gait speed improvement during the first 60 days poststroke: minimal clinically important difference. Phys Ther. 2010;90:196-208.

38. Binder SA, Moll CB, Wolf SL. Evaluation of electromyographic biofeedback as an adjunct to therapeutic exercise in treating the lower extremities of hemiplegic patients. Phys Ther. 1981;61:886-93.

39. Perera S, Mody SH, Woodman RC, Studenski SA. Meaningful change and responsiveness in common physical performance measures in older adults. J Am Geriatr Soc. 2006;54:743-9.

40. Gladstone DJ, Danells CJ, Black SE. The Fugl-Meyer assessment of motor recovery after stroke: a critical review of its measurement properties. Neurorehab Neural Re. 2002;16:232-40.

41. Flansbjer UB, Holmbäck AM, Downham D, Patten C, Lexell J. Reliability of gait performance tests in men and women with hemiparesis after stroke. J Rehabil Med. 2005;37:75-82

42. Nenadic Z. Information discriminant analysis: feature extraction with an information-theoretic objective. IEEE Trans Pattern Anal Mach Intell. 2007;29:1394-407.

43. Weir JP. Quantifying test-retest reliability using the intraclass correlation coefficient and the SEM. J Strength Cond Res. 2005;19:231-40.

44. Shrout PE, Fleiss JL. Intraclass Correlations: Uses in Assessing Rater Reliability. Psychol Bull. 1979;86:420-8.

45. Collen FM, Wade DT, Bradshaw CM. Mobility after stroke: reliability of measures of impairment and disability. Int Disabil Stud. 1990;12:6-9.

46. Hyndman D, Ashburn A, Stack E. Fall events among people with stroke living in the community: circumstances of falls and characteristics of fallers. Arch Phys Med Rehab. 2002:83:165-70.

47. Mackintosh SF, Hill K, Dodd KJ, Goldie P, Culham E. Falls and injury prevention should be part of every stroke rehabilitation plan. Clin Rehabil. 2005;19:441-51.

48. Jørgensen L, Engstad T, Jacobsen BK. Higher incidence of falls in long-term stroke survivors than in population controls: depressive symptoms predict falls after stroke. Stroke. 2002;33:542-7.

49. Righetti L, Buchli J, ljspeert AJ. Dynamic hebbian learning in adaptive frequency oscillators. Physica D. 2006;216:269-81.

50. Gomez-Rodriguez M, Grosse-Wentrup M, Hill J, Gharabaghi A, Scholkopf B, Peters J. Towards brain-robot interfaces in stroke rehabilitation, 12th Biann Int Conf on Rehabil Robot. 2011. p. 1-6.
51. Severinsen K, Jakobsen JK, Overgaard K, Andersen H. Normalized Muscle Strength, Aerobic Capacity, and Walking Performance in Chronic Stroke: A Population-Based Study on the Potential for Endurance and Resistance Training. Arch Phys Med Rehab. 2011;92:1663-8.

52. Little VL, McGuirk TE, Patten C. Impaired limb shortening following stroke: what's in a name? PLoS One. 2014;16:e110140.

\section{Submit your next manuscript to BioMed Central and take full advantage of:}

- Convenient online submission

- Thorough peer review

- No space constraints or color figure charges

- Immediate publication on acceptance

- Inclusion in PubMed, CAS, Scopus and Google Scholar

- Research which is freely available for redistribution 